\title{
openheart Effects of paediatric HIV infection on electrical conduction of the heart
}

\author{
Nikmah S Idris, ${ }^{1,2,3}$ Michael M H Cheung, ${ }^{3}$ Diederick E Grobbee, ${ }^{2}$ \\ David Burgner, ${ }^{3,4}$ Nia Kurniati, ${ }^{1}$ Mulyadi M Djer, ${ }^{1}$ Cuno S P M Uiterwaal ${ }^{2}$
}

To cite: Idris NS,

Cheung MMH, Grobbee DE, et al. Effects of paediatric HIV infection on electrical conduction of the heart. Open Heart 2016;3:e000340 doi:10.1136/openhrt-2015000340

Received 21 September 2015 Revised 15 December 2015 Accepted 20 December 2015

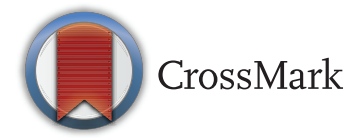

For numbered affiliations see end of article.

Correspondence to Dr Nikmah Salamia Idris; nikmah@ikafkui.org

\section{ABSTRACT}

Objective: To investigate the effects of HIV infection in children on heart electrical conduction, particularly to delineate the effects of HIV infection from treatment.

Methods: On a 12-lead ECG, available for 37 antiretroviral therapy (ART) naïve, 42 ART-exposed vertically-acquired HIV-infected and 50 healthy children in Jakarta, Indonesia, we measured cardiac conduction parameters: PR, QRS, and QTC (corrected using Bazett's formula) intervals. The associations between HIV infection/ treatment status and ECG intervals were evaluated using general linear modelling with further adjustment for potential confounders or intermediary variables. Findings are presented as (adjusted) mean differences between each of the two HIV groups and healthy children.

Results: Although not exceeding the clinical threshold for long QT (QTC >460 ms for girls and $>440 \mathrm{~ms}$ for boys) compared to healthy children, mean QTc intervals were longer in ART-naïve (difference $18.2 \mathrm{~ms}, 95 \% \mathrm{Cl}$ 7.0 to 29.3) and, to greater extent, in ART-exposed HIV-infected children (difference $28.9 \mathrm{~ms}, 19.3$ to 38.5). Following adjustment for RR interval, age and height, prolongation of $\mathrm{PR}$ interval was seen only in ART-naïve HIV-infected children (difference $12.9 \mathrm{~ms}, 2.4$ to 23.3). Cardiac mass/function, high-sensitive $\mathrm{C}$ reactive protein, cholesterol and glycated haemoglobin levels, systolic and diastolic blood pressures, or postnatal parental smoking exposure did not affect these associations. No difference in the QRS interval was observed between groups.

Conclusions: Prolongation of the QTC interval occurs in ART-naïve HIV-infected children and, to a greater extent, in the ART-exposed children, whereas a longer PR interval appears to be seen only among ART-naïve HIVinfected children.

\section{INTRODUCTION}

Vertically acquired HIV infection is a devastating disease in childhood which may affect any organs, including the cardiovascular system. ${ }^{1}$ Cardiac involvement may have diverse manifestations from initial asymptomatic presentation to overt heart failure to sudden death. ${ }^{2}$ With increased longevity of HIV-infected children, this chronic noninfectious complication becomes a confronting clinical challenge. ${ }^{3}$ There is an urgent need to better understand the impact of HIV infection on the developing cardiovascular

\section{KEY QUESTIONS}

What is already known about this patient?

- Autopsy has shown various pathologies in the cardiac conduction system of HIV-infected patients and ECG interval changes have been observed in adults. Paediatric data are very limited with no clear delineation between the effects of HIV infection and antiretroviral therapy (ART).

What does this study add?

- We showed that ART-naïve HIV infection is particularly associated with atrioventricular conduction (PR interval) prolongation, whereas ART exposure prolongs the QTc interval.

How might this impact on clinical practice?

- Since both QTc and PR interval changes may progress and become a substrate for fatal arrhythmia, routine ECG surveillance may be required for HIV-infected children.

system, including its underlying mechanisms, which most likely represent a complex interplay between HIV, antiretroviral therapy (ART), inflammation and co-infections. ${ }^{3}$ Among potential cardiovascular problems, conduction abnormalities have been rarely studied in HIV-infected children. ${ }^{4}{ }^{5}$ Autopsy showed that various pathologies may occur throughout the conduction system, including vasculitis, myocarditis or fragmentation of the His bundle with fibrosis. ${ }^{6}$ In adults, HIV infection is associated with a $20 \%$ prevalence of prolonged QTc interval, ${ }^{7}$ which potentially induces fatal ventricular arrhythmia, particularly torsade de pointes. ${ }^{8}$ A prolonged QTC interval also predicts mortality both in the healthy ${ }^{9}$ and high-risk populations, including those with previous myocardial infarction, diabetes mellitus ${ }^{10}$ or pulmonary hypertension. ${ }^{11}$ The development of QTc prolongation in HIV-infected adults may be associated with hyperlipidaemia, cardiac conditions, age, female sex, ethnicity, diabetes mellitus and perhaps ART, particularly the protease inhibitors. $^{12}$ 
Delineating the effects of HIV infection per se from ART on childhood cardiac conduction is difficult and has never been thoroughly studied. A US-based birth cohort $^{4}$ revealed QTc prolongation in treated and untreated HIV-infected children compared with HIV-exposed uninfected children; however, there was no clear disentanglement of the effects of HIV infection from ART, which is important from an aetiological point of view, as well as from a clinical perspective, as it may guide initial ART choices and ongoing follow-up as HIV-infected children transition to adulthood. Therefore, we aimed to investigate the effect of ART-naïve and ART-exposed HIV infection on cardiac electrical conduction in children.

\section{PATIENTS AND METHODS}

\section{Study design and population}

This was a cross-sectional analysis involving 114 vertically acquired HIV-infected (56 ART-naive and 58 ART-exposed) children and 51 healthy children enrolled in an ongoing cohort to evaluate cardiovascular complications of HIV infection in children. Between June 2013 and September 2014, HIV-infected children were recruited from the paediatric HIV clinics of Cipto Mangunkusumo National General Hospital, Koja District General Hospital and the Indonesian Planned Parenthood Association (IPPA), Jakarta, Indonesia. Healthy children of the same age range were enrolled from the same geographical area. We invited healthy children by directly approaching parents and community leaders and providing leaflets about the purpose and study procedures. Of the total of 55 children approached, 3 refused to participate and 1 was excluded due to suspected Down syndrome. We also excluded children with conditions suggestive of non-vertically acquired HIV (HIV-negative parents and a history of blood transfusion) as well as those with congenital or rheumatic heart diseases.
Of all included children, ECGs were available for 129 children, of whom 37 were ART-naïve, 42 ART-exposed, and 50 healthy children. Among the ART-naive children, five once received ART for $<3$ months but had been off treatment for at least 5 years prior to enrolment. Two of 42 ART-treated children had started treatment in our hospital for $<6$ months after being previously managed in another hospital with a potential compliance issue. Of 36 children who did not have the ECG measurements, 9 died (of whom 5 were ART-naïve HIV-infected children), 2 had a poor-quality recording, 3 moved out of town and, of those remaining, the children refused the examination (figure 1).

Ethical approval was obtained from the Ethics Committee, Faculty of Medicine University of Indonesia and University of Melbourne. Informed consent was obtained from all participating families.

\section{Exposure measurement}

We established the diagnosis of HIV infection on the basis of the WHO criteria. ${ }^{13}$ Briefly, in children aged $<18$ months, diagnosis was made by a positive HIV DNA or RNA PCR, whereas for those aged $>18$ months, diagnosis was confirmed by positive HIV serology. To measure disease severity, we further classified children on the basis of the WHO clinical and immunological staging. For clinical staging, we applied the criteria using patients' actual condition at the time of ECG measurement. The CD4 cell percentage, used to define immunological staging, was obtained from routine clinical data within 3 months of ECG measurement. Viral load examination was not routinely performed in our clinical setting due to financial constraints and is only performed as indicated by treating physicians.

\section{ECG measurement}

We performed 12-lead ECG examinations on children in the supine position with the speed set at $25 \mathrm{~mm} / \mathrm{s}$ and

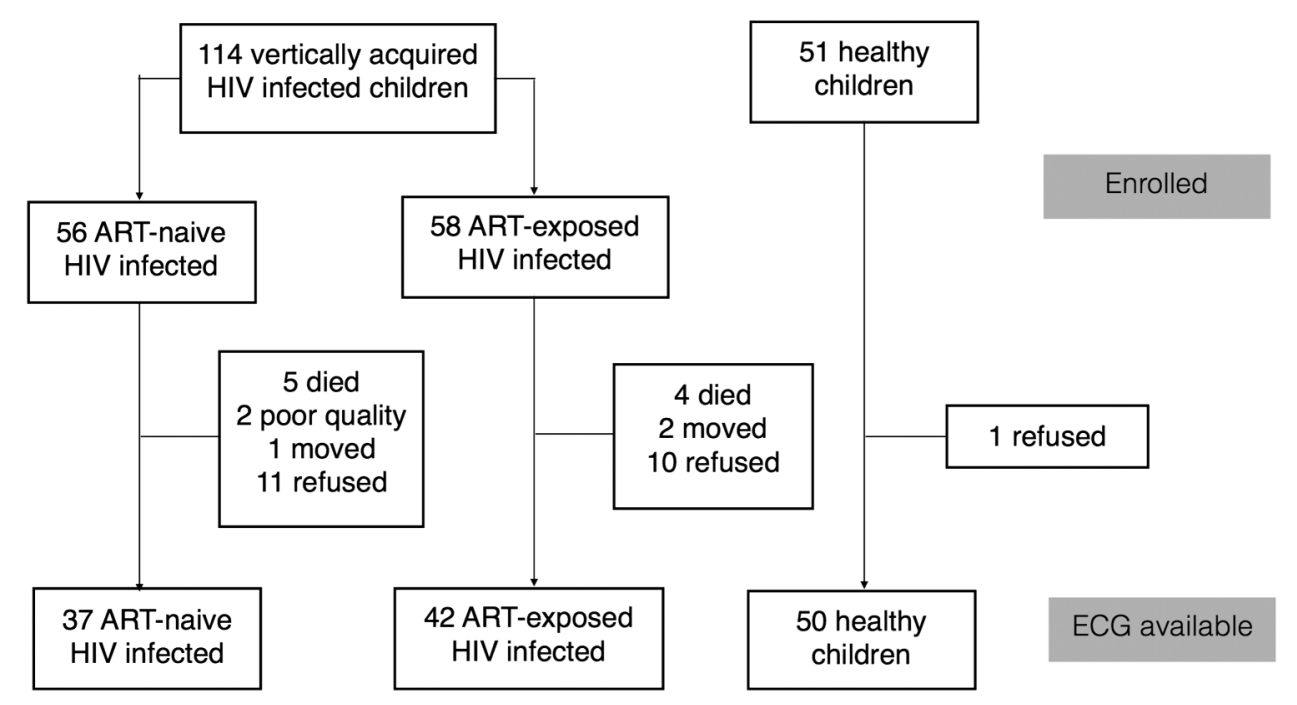

Figure 1 Flow chart of study participant. ART, antiretroviral therapy. 
the gain at $10 \mathrm{~mm} / \mathrm{mV}$. The ECG was interpreted manually using a calibrated ruler by a single investigator (NSI) blinded to HIV infection or treatment status. Twenty randomly selected recordings were separately measured by paediatric cardiologists (MMHC, MMD). Conduction (depolarisation and repolarisation) parameters, including PR, QRS and QT intervals, were measured as an average of three beats in lead II in most patients and in lead V5 in five patients due to low-voltage lead II. The PR interval, reflecting atrial and atrioventricular (AV) nodal depolarisation, was defined as the distance from the beginning of the $\mathrm{P}$ wave to the beginning of the first deflection of the QRS complex, and was measured in the shortest RR interval. The QT intervals, representing total depolarisation-repolarisation time, were measured after the shortest RR interval. The QT interval was defined as starting at the beginning of the first deflection of the QRS complex and lasting until the end of the T wave (QT). To determine the end of the QT interval, we applied the tangential method as described previously. ${ }^{14}$ The QT interval was corrected for heart rate using Bazett's formula $(\mathrm{QTc}=\mathrm{QT} / \sqrt{ } \mathrm{RR})$, while the $\mathrm{PR}$ interval was corrected for the RR interval in the statistical model. All interval measurements are presented in milliseconds.

Of the 129 ECGs available, 124 and 123 were analysable for QT and PR intervals, respectively. Five ECGs were not analysable for QT interval due to the right bundle branch block and non-specific ST/T wave changes, whereas six ECGs were not analysable for PR interval measurement due to the low-voltage $\mathrm{P}$ wave.

\section{Measurement of potential confounders and intermediary variables}

We a priori included age and body mass index (BMI) as potential confounders in the association between HIV infection status and cardiac conduction. In addition to their association with HIV infection status, both variables are most likely related to cardiac conduction time, to which some associations have been reported previously. ${ }^{15}$ Since cardiac conditions (mass and function) ${ }^{16}$ as well as inflammation may mediate the effect of ART-naïve or ART-exposed HIV infection on heart conduction parameters, we considered left ventricular (LV) mass, ejection fraction and high-sensitive $\mathrm{C}$ reactive protein (hs-CRP) level as possible intermediary variables. LV mass index and ejection fraction were measured by echocardiographic M-mode and Biplane Simpson's methods, respectively, using standard techniques. ${ }^{17}$ In addition, since dyslipidaemia, diabetes, blood pressure and smoking exposure have been reported to be associated with ECG intervals, including in HIV-infected populations ${ }^{18}$ we also explored the possible intermediary roles of these variables on the associations between ART-naive or ART-exposed HIV infection in children and cardiac conduction.

Data on age and smoking exposure were obtained using a standard questionnaire filled by parents or caregivers, whereas BMI was calculated from weight and height obtained by standard measurements. Laboratory values, including lipid, glycated haemoglobin (HbAlc) and hs-CRP levels, were analysed at Cipto Mangunkusumo Clinical Pathology Laboratory, Jakarta. Since some medications have been implicated in the ECG interval abnormalities, ${ }^{19}$ we also recorded medication use at the time of assessment. On the basis of previous literature, we considered macrolides, trimethoprim, azole antifungals, antihistamine ${ }^{19}$ and protease inhibitors $^{20}$ to potentially prolong the QTC interval.

\section{Data analysis}

We described baseline characteristics as mean, median or proportion, as appropriate, based on child HIV status. The distributions of categorical and numerical variables across the HIV status groups were statistically evaluated using the $\chi^{2}$ and one-way analysis of variance (ANOVA), or Kruskal-Wallis tests, respectively.

To investigate the effects of HIV infection on heart conduction, we performed univariable and multivariable general linear modelling with HIV status as a fixed factor and ECG interval parameters (PR, QRS and QTc intervals) as separate dependent variables. The same models were used for adjustment for age and BMI as potential confounders. For the PR interval, we initially adjusted for the RR interval before adjusting for potential confounders. To explore the role of various potential intermediary variables, we made graphical presentations of the effects of adding each of these variables into the confounder-adjusted model. A graphical presentation was also made to explore the influence of a particular type of drugs on the QTc interval in ART-exposed children as well as ART-naïve HIV-infected children. We also performed a sensitivity analysis by excluding five ART-naive HIV-infected children who once received a brief period of ART in the past.

Findings are presented as crude and adjusted mean differences with $95 \%$ CIs and corresponding $\mathrm{p}$ values. A 95\% CI not including zero, corresponding to a $p$ value of $<0.05$, was considered statistically significant. All statistical analyses were performed using SPSS V.19 and RStudio 0.98.507 for Mac.

\section{RESULTS}

Baseline characteristics of study patients are described in table 1. Children with HIV infection, both naïve or exposed to ART, came from a lower socioeconomic status and were more heavily exposed to both prenatal and postnatal parental smoking. ART-naïve HIV-infected patients were younger and had a smaller body size and the lowest LV mass. Despite similar BMI with healthy children, the ART-exposed HIV-infected group had the highest LV mass. With respect to cardiac function, HIV-infected children, both ART-naïve and ART-exposed, tended to have a lower LV ejection fraction than the healthy ones. 
Table 1 Baseline characteristics of study participants

\begin{tabular}{|c|c|c|c|c|}
\hline Characteristics & ART-naïve HIV n=37 & ART-exposed HIV n=42 & Non-HIV $n=50$ & p Value \\
\hline Age $\left(\right.$ years) ${ }^{\star}$, median (range) & $4.8(0.4-12.5)$ & $6.1(1.3-12.2)$ & $6.4(2.4-14.0)$ & 0.22 \\
\hline Male gender, n (\%) & $15(41)$ & $21(50)$ & $23(46)$ & 0.70 \\
\hline Low socioeconomic, n (\%) & $16(44)$ & $25(60)$ & $10(20)$ & 0.001 \\
\hline \multicolumn{5}{|l|}{ Parental smoking after birth, $\mathrm{n}(\%)$} \\
\hline Mother & $3(9)$ & $5(12)$ & $0(0)$ & 0.05 \\
\hline Father/other household member & $30(81)$ & $35(83)$ & $33(66)$ & 0.11 \\
\hline \multicolumn{5}{|l|}{ Parental smoking in pregnancy, $\mathrm{n}(\%)$} \\
\hline Mother & $6(18) \dagger$ & $7(18)$ & $1(2.0)$ & 0.03 \\
\hline Father/other household member & $29(78)$ & $34(81)$ & $34(68)$ & 0.31 \\
\hline Body weight $(\mathrm{kg})$ & $14.7(8.2)$ & $18.8(5.4)$ & $22.4(9.6)$ & $<0.001$ \\
\hline Body height (cm) & $99.2(21.9)$ & $109.1(14.0)$ & $116.3(17.8)$ & $<0.001$ \\
\hline Body mass index $\left(\mathrm{kg} / \mathrm{m}^{2}\right)$ & $14.2(2.2)$ & $15.5(1.9)$ & $15.9(2.8)$ & 0.01 \\
\hline Systolic BP (mm Hg) & $101.6(10.7)$ & $99.6(9.7)$ & $102.4(9.7)$ & 0.41 \\
\hline Diastolic BP (mm Hg) & $63.1(9.7)$ & $60.4(7.5)$ & $61.3(7.4)$ & 0.37 \\
\hline Total cholesterol (mmol/L) & $3.3(0.9)$ & $4.5(0.9)$ & $4.4(0.7)$ & $<0.001$ \\
\hline LDL cholesterol (mmol/L) & $1.9(0.7)$ & $2.6(0.7)$ & $2.6(0.6)$ & $<0.001$ \\
\hline $\mathrm{HbA1c}$ level & $0.05(0.04-0.06)$ & $0.05(0.04-0.07)$ & $0.06(0.04-0.07)$ & 0.30 \\
\hline hs-CRP, median (range), nmol/L & $36(0-2759)$ & 47 (3.7-1227) & $4.7(0-155)$ & $<0.001 \ddagger$ \\
\hline HIV clinical stage 3 or 4 & $30(85.7) \S$ & $18(47.4)$ & & $<0.001$ \\
\hline CD4 absolute level $(n=56)$ & $258(8-2716)$ & $1183(4-2301)$ & - & $<0.001$ \\
\hline CD4\% $(n=52)$ & $10(1-41)$ & 30 (4-39) & - & 0.001 \\
\hline Viral load $(n=19)$ & $5840\left(220-2.0 \times 10^{5}\right)$ & $185\left(0-2.1 \times 10^{6}\right)$ & & \\
\hline LV EF (\%), median (range) & $64.5(24.8-80.5)$ & $63.1(39.3-82.4)$ & $67.4(58.6-80.9)$ & 0.04 \\
\hline LV mass $(g)$ & $41.9(16.6)$ & $61.1(18.6)$ & $49.8(17.3)$ & 0.001 \\
\hline Use of QT prolonging drugs & $16(43.2)$ & $20(34.5)$ & $0(0)$ & \\
\hline ART use in pregnancy, $n$ (\%) & $0(0)$ & $0(0)$ & $0(0)$ & \\
\hline Pulse rate (bpm) & $124(26)$ & $119(22)$ & $115(19)$ & 0.14 \\
\hline QTc interval (milliseconds), $n=124$ & $402.9(25.7)$ & $415.0(23.7)$ & $388.9(20.7)$ & ** \\
\hline PR interval (milliseconds), $n=123$ & $131.0(25.4)$ & $126.1(24.9)$ & $122.7(19.7)$ & ** \\
\hline QRS interval (milliseconds), $n=125$ & $68.2(17.3)$ & $65.7(10.5)$ & $66.1(11.4)$ & ** \\
\hline \multicolumn{5}{|c|}{$\begin{array}{l}\text { †Percentage based on } 34 \text { ART-naïve HIV-infected patients. } \\
\text { †Kruskal-Wallis test. } \\
\text { SPercentage based on } 35 \text { ART-naïve HIV-infected patients. } \\
\text { १९Macrolides, trimethoprim, azole antifungals, antihistamine and protease inhibitors. } \\
{ }^{*} \text { Otherwise stated, data is presented as mean (SD). } \\
\text { **See table } 2 \text { for statistical analysis. } \\
\text { ART, antiretroviral therapy; BP, blood pressure; EF, ejection fraction; HbA1c, glycated haemoglobin; hs-CRP, high-sensitive C reactive protein; } \\
\text { LDL, low-density lipoprotein; LV, left ventricular. }\end{array}$} \\
\hline
\end{tabular}

ART-exposed HIV-infected children had the highest low-density lipoprotein (LDL) cholesterol and lower high-density lipoprotein levels compared with healthy children; however, no statistically significant difference in HbA1c level was observed between the groups. With regard to disease severity, ART-naive HIV-infected children were in more severe clinical and immune deficient stages than the ART-exposed children. The median duration of treatment in the ART-exposed group was 2.4 (0.3-9.9) years. Only five ART-exposed HIV-infected children received a regimen containing protease inhibitor drugs. At the time of ECG measurement, around one-third of ART-naïve and ART-exposed HIV-infected children took medications known to potentially affect the QTc interval.

Although the mean QTc and PR intervals of ART-naïve and ART-exposed HIV-infected children (table 1) were not beyond the clinically acceptable thresholds for the long QT syndrome and first-degree AV block, ${ }^{21}$ respectively, there was an increase towards abnormality compared with the normal control. Table 2 summarises the effects of ART-naive or ART-exposed HIV infection on these cardiac electrical conduction parameters. Compared to healthy children, QTc intervals were longer in ART-naïve and, to greater extent, in ART-exposed HIV-infected children as shown in the crude (mean difference $14.0(\mathrm{p} \leq 0.001)$ and $26.2 \mathrm{~ms}$ $(\mathrm{p}<0.001)$, respectively) as well as final adjusted analysis (difference $18.3 \quad(\mathrm{p} \leq 0.001)$ and $28.9 \mathrm{~ms} \quad(\mathrm{p}<0.001)$, respectively). These associations could not be explained by cardiac mass/function, hs-CRP level, lipid and HbAlc levels, systolic and diastolic blood pressures, nor by postnatal parental smoking exposure (figure 2). There were six children (1 ART-naïve and 5 ART-exposed) who had QTc intervals of $>470 \mathrm{~ms}$. In terms of the PR interval, prolongation was seen only in ART-naïve HIV-infected 
Figure 2 Influence of possible intermediary variables on QTc interval mean difference $(95 \% \mathrm{Cl})$ between HIV-infected (ART-naïve or ART-exposed) children and healthy children. ART, antiretroviral therapy; DBP, diastolic blood pressure; EF, ejection fraction; HbA1c, glycated haemoglobin; hsCRP, high-sensitive $\mathrm{C}$ reactive protein; LDL, low-density lipoprotein; LV, left ventricular; SBP, systolic blood pressure.

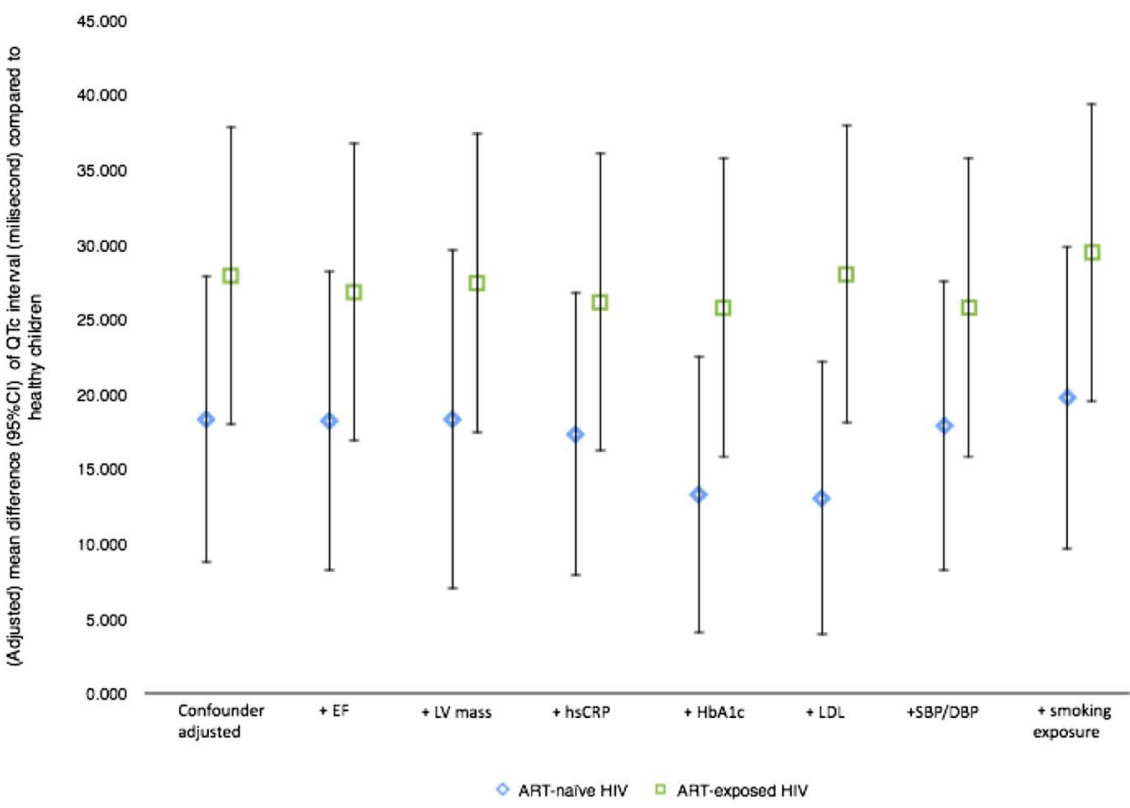

children after adjustment for RR interval, age and height (difference $=12.9 \mathrm{~ms}, \mathrm{p}=0.02$ ). This finding was not substantially explained by cardiac conditions, hs-CRP, LDL cholesterol, or HbA1c levels, blood pressures, or parental smoking exposure (figure 3). Analysis restricted to 32 children who had never received any ART did not substantially affect the results (table 2).

The influence of drugs known to be associated with QT prolongation among HIV-infected children is shown in figure 4. In the ART-exposed HIV-infected group, children who used protease inhibitors tended to have longer QTc intervals compared with those who did not, whereas the use of other QT prolongation drugs seemed to have little and inconsistent effect in the ART-exposed and ART-naïve HIV-infected patients. No statistical association was explored due to the limited numbers of children who used protease inhibitors or other QT prolongation drugs.

\section{DISCUSSION}

We demonstrated that HIV infection in children is associated with a longer QTc interval both in ART-naïve and, to a greater extent, in ART-exposed children, compared with uninfected controls. In ART-naïve HIV-infected children only, there was a longer PR interval. However, these prolongations in QTc and PR intervals were not above the clinically acceptable thresholds for abnormality. Neither cardiac conditions, degree of inflammation, lipid or HbAlc levels, blood pressure, nor parental smoking exposures appeared to mediate these associations.

To the best of our knowledge, this is the first study to delineate the effects of ART-naive from ART-exposed paediatric HIV infection on cardiac electrical conduction. A previous larger study ${ }^{4}$ with $24 \mathrm{~h}$ Holter ECG monitoring did not differentiate the possible effects of ART despite showing similar results of QTc interval prolongation in HIV-infected children compared with HIV-exposed uninfected children. Differentiating the effects of HIV infection per se from treatment is important from aetiological and clinical perspectives as it may have repercussions on treatment strategies. We demonstrated differences in the effects of ART-treated and ART-untreated HIV infection in children on heart conduction. Even though they shared common effects on QTc prolongation, the magnitude differed. Despite the novelty of this study, we were unable to obtain ECG measurements for all children enrolled in this study. Of these, almost one quarter was due to the death of the patients, particularly in the ART-naive HIV-infected group. We may therefore have unavoidably missed the effects of the most severe form of untreated HIV infection on the cardiac conduction, possibly resulting in an underestimation of the findings in the total population. In addition, with regard to our exploration for possible intermediary factors in the association between HIV infection and cardiac conduction, we did not have the opportunity to measure serum electrolytes. We did not perform a detailed assessment for the possibility of familial long QT syndrome in our subjects, which we believe to be exceedingly rare $(1$ in 2500$) .{ }^{22}$

In this study, we only performed standard ECG at a single time point rather than Holter ambulatory recordings. Although ambulatory monitoring would be particularly informative in capturing ECG interval variations and relating it to pharmacokinetic data, this was beyond the scope of our study. Moreover, in terms of conduction abnormalities, a standard ECG recording remains the cornerstone for diagnostic purposes and is the only practical option, especially in resource-limited settings. Holter monitoring has many potential problems, 


\begin{tabular}{|c|c|c|c|c|c|c|c|c|c|c|c|}
\hline & & & QRS interve & milliseconds) & & QTc interva & milliseconds) & & PR interval & illiseconds) & \\
\hline Model & $\begin{array}{l}\text { HIV/treatment } \\
\text { group }\end{array}$ & & $\begin{array}{l}\text { Mean } \\
\text { difference }\end{array}$ & $95 \% \mathrm{Cl}$ & $p$ Value & $\begin{array}{l}\text { Mean } \\
\text { difference }\end{array}$ & $95 \% \mathrm{Cl}$ & & $\begin{array}{l}\text { Mean } \\
\text { difference }\end{array}$ & $95 \% \mathrm{Cl}$ & $p$ Value \\
\hline \multirow[t]{4}{*}{ Crude } & Non-HIV & & Ref & & & Ref & & & Ref & & \\
\hline & ART-naïve HIV & All 37 & 2.9 & -2.6 to 8.3 & 0.31 & 14.0 & 3.8 to 2.3 & $<0.001$ & 8.3 & -2.1 to 18.6 & 0.12 \\
\hline & & $\begin{array}{l}32 \text { Never } \\
\text { ART }\end{array}$ & 2.5 & -3.2 to 8.1 & 0.39 & 11.5 & 0.4 to 22.5 & 0.04 & 4.7 & -6.1 to 15.5 & 0.39 \\
\hline & $\begin{array}{l}\text { ART-exposed } \\
\text { HIV }\end{array}$ & & -0.4 & -5.7 to 4.8 & 0.90 & 26.2 & 16.7 to 35.6 & $<0.001$ & 3.4 & -6.1 to 12.9 & 0.49 \\
\hline RR interval & Non-HIV & & & & & & & & $\operatorname{Ref}^{*}$ & & \\
\hline \multirow[t]{3}{*}{ adjustment } & ART-naïve HIV & All 37 & & & & & & & 9.2 & -1.1 to 19.6 & 0.08 \\
\hline & & $\begin{array}{l}32 \text { Never } \\
\text { ART }\end{array}$ & & & & & & & 6.7 & -4.3 to 17.5 & 0.23 \\
\hline & $\begin{array}{l}\text { ART-exposed } \\
\text { HIV }\end{array}$ & & & & & & & & 4.1 & -5.4 to 13.6 & 0.40 \\
\hline \multirow[t]{4}{*}{ Model 1} & Non-HIV & & Ref & & & Ref & & & Ref $†$ & & \\
\hline & ART-naïve HIV & All 37 & 4.6 & -0.8 to 10.1 & 0.10 & 16.9 & 6.7 to 27.1 & $<0.001$ & 10.5 & -0.03 to 21.0 & 0.05 \\
\hline & & $\begin{array}{l}32 \text { Never } \\
\text { ART }\end{array}$ & 5.2 & -0.7 to 11.0 & 0.09 & 15.7 & 4.5 to 27.0 & $<0.001$ & 7.8 & -3.6 to 19.2 & 0.18 \\
\hline & $\begin{array}{l}\text { ART-exposed } \\
\text { HIV }\end{array}$ & & 0.1 & -5.0 to 5.2 & 0.97 & 27.1 & 17.8 to 36.3 & $<0.001$ & 4.2 & -5.3 to 13.9 & 0.42 \\
\hline \multirow[t]{4}{*}{ Model 2} & Non-HIV & & & & & Ref & & & Ref $\ddagger$ & & \\
\hline & ART-naïve HIV & All 37 & 5.0 & -1.0 to 11.0 & 0.10 & 18.2 & 7.0 to 29.3 & $<0.001$ & 12.9 & 2.4 to 23.3 & 0.02 \\
\hline & & $\begin{array}{l}32 \text { Never } \\
\text { ART }\end{array}$ & 4.6 & -2.0 to 11.1 & 0.17 & 17.0 & 4.7 to 29.3 & $<0.001$ & 13.3 & 1.6 to 24.9 & 0.03 \\
\hline & $\begin{array}{l}\text { ART-exposed } \\
\text { HIV }\end{array}$ & & 0.2 & -5.1 to 5.5 & 0.94 & 28.9 & 19.3 to 38.5 & $<0.001$ & 4.3 & -4.7 to 13.3 & 0.35 \\
\hline $\begin{array}{l}\text { Model 1: adju } \\
{ }^{*} \text { Adjusted for } \\
\text { †Model } 1+\mathrm{ac} \\
\text { †Model } 2+\mathrm{ac} \\
\text { ART, antiretro }\end{array}$ & $\begin{array}{l}\text { or age; model 2: adju } \\
\text { erval. } \\
\text { d for RR interval. } \\
\text { dfor RR interval. } \\
\text { eerapy; BMI, body m }\end{array}$ & $\begin{array}{l}\text { d for age, BI } \\
\text { index. }\end{array}$ & & & & & & & & & \\
\hline
\end{tabular}

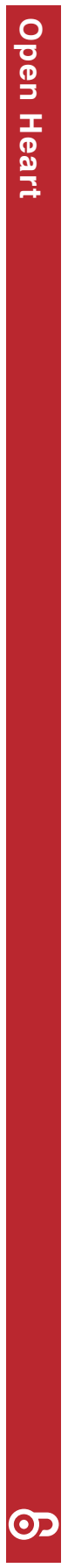

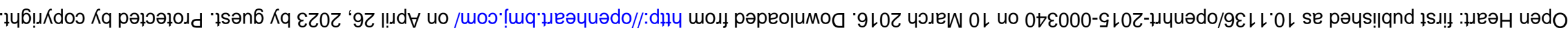


Figure 3 Influence of possible intermediary variables on PR interval mean difference $(95 \% \mathrm{Cl})$ between HIV-infected (ART-naïve or ART-exposed) children and healthy children. ART, antiretroviral therapy; DBP, diastolic blood pressure; EF, ejection fraction; HbA1c, glycated haemoglobin; hsCRP, high-sensitive $C$ reactive protein; LDL, low-density lipoprotein; LV, left ventricular; SBP, systolic blood pressure.

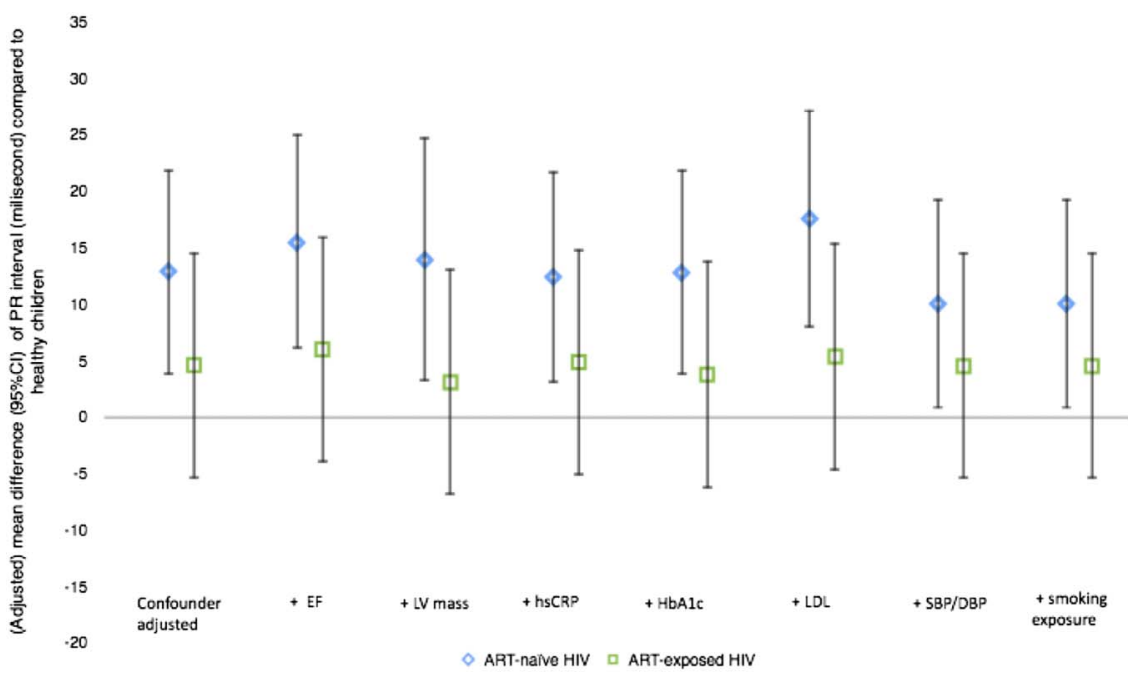

including changes in ECG morphology and multiple artefacts due to postural variations or motion. ${ }^{23}$ In addition, the added value of QT interval variability recorded by ambulatory ECG in predicting significant ventricular arrhythmia remains controversial. ${ }^{24}$

We found QTc prolongation in ART-naïve children and, to a greater degree, in ART-exposed HIV-infected children. In the setting of a similar QRS interval with healthy children, this indicates that HIV-infected children may have prolonged repolarisation. On the basis of these findings, we may infer that HIV infection per se, independent of age and nutritional status, does have some effects on QTc interval and ART or the interaction between ART and the disease is likely to augment these effects. Prolongation of the QTc interval has also been reported in HIV-infected adults and is suggested to be associated with HIV infection duration. ${ }^{25}$ However, since our finding remained after adjusting for age, which in children reflects the duration of vertically-acquired HIV infection, the effects in children may be independent of infection duration. Furthermore, since these associations are not explained by cardiac conditions, inflammation, plasma lipids, blood pressure or smoking exposure, HIV

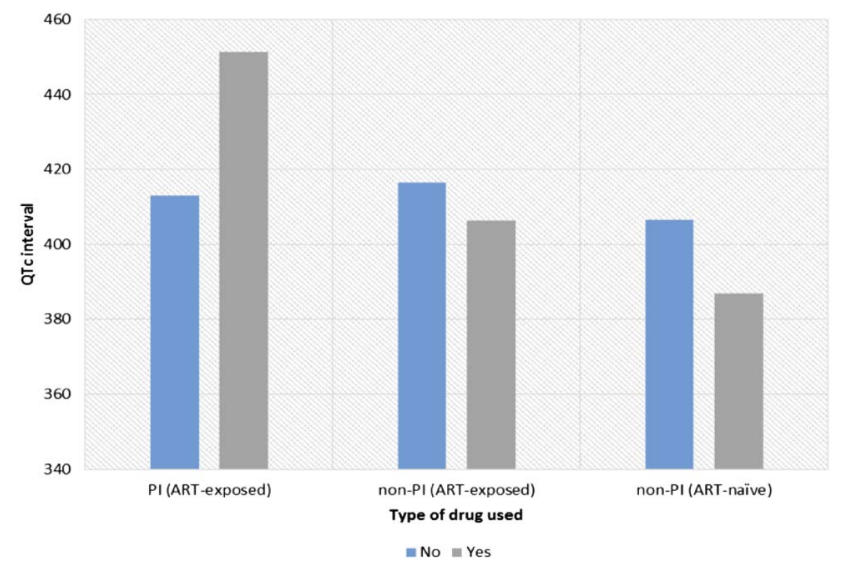

Figure 4 QTc interval based on the use of QT-prolonging drugs in HIV-infected children. ART, antiretroviral therapy. infection or its treatment may have a direct effect on the autonomic cardiac regulation or conduction pathway, although the possibility of electrolyte abnormalities should also be considered. A recent animal study showed that an HIV-1 transactivator of transcription (Tat, fragment 1-86) induced an increase in cytosolic calcium concentration in cardiac-projecting parasympathetic neurons of nucleus ambiguus, triggering prolonged bradycardia, which consequently may be we also found greater prolongation of the QT interval in ART-exposed HIV-infected children, despite a low rate of use of protease inhibitors, warrants further exploration of the effects of non-protease inhibitor drugs on the cardiac conduction system, in interaction with HIV infection.

We found that ART-naïve HIV-infected children had longer PR intervals than healthy children, which has not been reported previously. A longer PR interval may indicate increased predisposition to first-degree $\mathrm{AV}$ block and is associated with a $20-30 \%$ increased risk of atrial fibrillation, ${ }^{27}$ despite controversies regarding its prognostic significance in the healthy general population. ${ }^{28}$ In our ART-naive HIV-infected cohort, the occurrence of a longer PR interval may be associated with underlying cardiac pathology or pulmonary hypertension causing heart atria dilation; unfortunately, we did not measure atrial size in this study.

Although the mean values of QTc and PR intervals in our HIV-infected study population are still below the various cut-off values for congenital long QT syndrome in children (QTc interval of $450-480 \mathrm{~ms})^{21}$ or for firstdegree AV block, our findings may be of clinical importance. First, some individual HIV-infected children in our cohort reached these thresholds. Furthermore, in HIV-infected children, the association between longer QTc and PR intervals with clinical outcomes, such as neurological manifestations, mortality or sudden death, warrants further investigation. As demonstrated by ECG findings in HIV-infected adults, our findings may reflect involved in the QT interval prolongation. ${ }^{26}$ The fact that 
an initial pathological process that progresses as the duration of HIV infection and treatment increases, so that routine ECG surveillance may be beneficial for HIV-infected children.

In conclusion, both ART-naïve and, to a greater extent, ART-exposed HIV infections in children are associated with prolongation of the QTc interval, whereas a longer PR interval only occurs in ART-naïve HIV-infected children. These associations are unlikely to be mediated by cardiac structural abnormality or systolic function, level of inflammation, lipid or glucose metabolism, blood pressures, nor by parental smoking exposures.

\section{Author affiliations}

${ }^{1}$ Faculty of Medicine University of Indonesia-Cipto Mangunkusumo Hospital, Department of Child Health, Jakarta, Indonesia

${ }^{2}$ Julius Global Health, Julius Centre for Health Sciences and Primary Care, the University Medical Centre Utrecht, Utrecht, The Netherlands

${ }^{3}$ Department of Paediatrics, University of Melbourne, Murdoch Childrens

Research Institute; Royal Children's Hospital, Melbourne, Australia

${ }^{4}$ Department of Paediatrics, Monash University, Victoria, Australia

Acknowledgements The authors gratefully acknowledge Septiani Madonna Gultom, Hanitya Dwi Ratnasari (the deceased), Weni Tenlima and Gina Puspita for their dedicated assistance in data collection, Diah Kristanty for performing the laboratory assays, and Rik Hopmans, MSc (Eindhoven University of Technology) for assistance with the R statistical programme. They also thank the medical/nursing staff of Cipto Mangunkusumo and Koja hospitals; Edi Sugiarto, Sri Yani and Santi Sardi from the Indonesian Planned Parenthood Association Clinic (an associate member of International Planned Parenthood Federation); all patients and families for their invaluable contribution in this project.

Contributors NSI contributed to the study conception/design, data collection, data analysis and interpretation, and manuscript writing. MMHC and DEG contributed to the study conception/design, data interpretation, and critical intellectual input for manuscript content. DB and NK contributed to the study conception/design and critical intellectual input for manuscript content. MMD contributed to the data interpretation and critical intellectual input for manuscript content. CSPMU contributed to the study conception/design, data analysis and interpretation, data interpretation, and manuscript writing.

Competing interests None declared.

Patient consent Obtained.

Ethics approval Ethics Committee of Faculty of Medicine University of Indonesia.

Provenance and peer review Not commissioned; externally peer reviewed.

Data sharing statement No additional data are available.

Open Access This is an Open Access article distributed in accordance with the Creative Commons Attribution Non Commercial (CC BY-NC 4.0) license, which permits others to distribute, remix, adapt, build upon this work noncommercially, and license their derivative works on different terms, provided the original work is properly cited and the use is non-commercial. See: http:// creativecommons.org/licenses/by-nc/4.0/

\section{REFERENCES}

1. Sims A, Hadigan C. Cardiovascular complications in children with HIV infection. Curr HIV/AIDS Rep 2011;8:209-14

2. Idris NS, Grobbee DE, Burgner D, et al. Cardiovascular manifestations of HIV infection in children. Eur J Prev Cardiol 2015;22:1452-61.

3. Shah MR, Cook N, Wong R, et al. Stimulating high impact HIV-related cardiovascular research: recommendations from a multidisciplinary NHLBI Working Group on HIV-related heart, lung, and blood disease. J Am Coll Cardiol 2015;65:738-44.
4. Saidi AS, Moodie DS, Garson A Jr, et al. Electrocardiography and 24-hour electrocardiographic ambulatory recording (Holter monitor) studies in children infected with human immunodeficiency virus type 1. The Pediatric Pulmonary and Cardiac Complications of Vertically Transmitted HIV-1 Infection Study Group. Pediatr Cardiol 2000;21:189-96.

5. Benchimol-Barbosa PR. Circadian cardiac autonomic function in perinatally HIV-infected preschool children. Braz J Med Biol Res 2009;42:722-30.

6. Bharati S, Joshi VV, Connor EM, et al. Conduction system in children with acquired immunodeficiency syndrome. Chest 1989;96:406-13.

7. Sani MU, Okeahialam BN. QTc interval prolongation in patients with HIV and AIDS. J Natl Med Assoc 2005;97:1657-61.

8. Kocheril AG, Bokhari SA, Batsford WP, et al. Long QTc and torsades de pointes in human immunodeficiency virus disease. Pacing Clin Electrophysiol 1997;20:2810-16.

9. Lindekleiv $\mathrm{H}$, Wilsgaard T, Macfarlane PW, et al. QT interval and the risk of myocardial infarction and all-cause death: a cohort study. J Cardiovasc Electrophysiol 2012;23:846-52.

10. Cox AJ, Azeem A, Yeboah J, et al. Heart rate-corrected QT interval is an independent predictor of all-cause and cardiovascular mortality in individuals with type 2 diabetes: the Diabetes Heart Study. Diabetes Care 2014;37:1454-61.

11. Rich JD, Thenappan T, Freed B, et al. QTc prolongation is associated with impaired right ventricular function and predicts mortality in pulmonary hypertension. Int J Cardiol 2013;167: 669-76.

12. Singh P, Hemal A, Agarwal S, et al. Cardiac manifestations in HIV infected children. Indian J Pediatr 2015;82:230-4.

13. World Health Organization. WHO case definitions of HIV for surveillance and revised clinical staging and immunological classification of HIV-related disease in adults and children. Geneva: WHO Press, 2007.

14. Lepeschkin E, Surawicz B. The measurement of the Q-T interval of the electrocardiogram. Circulation 1952;6:378-88.

15. Nasir JM, Rubal BJ, Jones SO, et al. The effects of body mass index on surface electrocardiograms in young adults. $J$ Electrocardiol 2012;45:646-51.

16. Soliman EZ, Shah AJ, Boerkircher A, et al. Inter-relationship between electrocardiographic left ventricular hypertrophy and QT prolongation as predictors of increased risk of mortality in the general population. Circ Arrhythm Electrophysiol 2014;7:400-6.

17. Lopez L, Colan SD, Frommelt PC, et al. Recommendations for quantification methods during the performance of a pediatric echocardiogram: a report from the pediatric measurements writing group of the American society of echocardiography pediatric and congenital heart disease council. J Am Soc Echocardiogr 2010;23:465-95.

18. Reinsch N, Buhr C, Krings $\mathrm{P}$, et al. Prevalence and risk factors of prolonged QTc interval in HIV-infected patients: results of the HIV-HEART study. HIV Clin Trials 2009;10:261-8.

19. Yap YG, Camm AJ. Drug induced QT prolongation and torsades de pointes. Heart 2003;89:1363-72.

20. Anson BD, Weaver JG, Ackerman MJ, et al. Blockade of HERG channels by HIV protease inhibitors. Lancet 2005;365:682-6.

21. Hofman N, Wilde AA, Kääb $S$, et al. Diagnostic criteria for congenita long QT syndrome in the era of molecular genetics: do we need a scoring system? Eur Heart J 2007;28:575-80.

22. Johnson JN, Ackerman MJ. QTc: how long is too long? Br J Sports Med 2009;43:657-62.

23. Christiansen JL, Guccione P, Garson A Jr. Difference in QT interval measurement on ambulatory ECG compared with standard ECG. Pacing Clin Electrophysiol 1996;19:1296-303.

24. Puljevic D, Smalcelj A, Durakovic Z, et al. QT dispersion, daily variations, QT interval adaptation and late potentials as risk markers for ventricular tachycardia. Eur Heart J 1997;18:1343-9.

25. Fiorentini A, Petrosillo N, Di Stefano A, et al. QTc interval prolongation in HIV-infected patients: a case-control study by 24-hour Holter ECG recording. BMC Cardiovasc Disord 2012;12:124.

26. Brailoiu E, Deliu E, Sporici RA, et al. HIV-1-Tat excites cardiac parasympathetic neurons of nucleus ambiguus and triggers prolonged bradycardia in conscious rats. Am J Physiol Regul Integr Comp Physiol 2014;306:R814-22.

27. Nielsen JB, Pietersen A, Graff C, et al. Risk of atrial fibrillation as a function of the electrocardiographic $P R$ interval: results from the Copenhagen ECG Study. Heart Rhythm 2013;10:1249-56.

28. Cheng S, Keyes MJ, Larson MG, et al. Long-term outcomes in individuals with prolonged PR interval or first-degree atrioventricular block. JAMA 2009;301:2571-7. 